\title{
Thrombocytopenia and low-dose heparin administration
}

\author{
THOMAS F. MORLEY, D.O., FCCP \\ Stratford, New Jersey \\ GREGORY BANNETT, D.O \\ Danville, Pennsylvania \\ TERESA MORONE, D.O \\ Wenonah, New Jersey \\ JAMES C. GIUDICE, D.O., FCCP \\ NATHAN FREED, D.O \\ Stratford, New Jersey
}

A prospective study was

conducted to determine the frequency of thrombocytopenia following the subcutaneous administration of low-dose heparin $(5,000$ units every 12 hours). Sixty-five patients were treated for an average of 11 days. Two of the patients developed thrombocytopenia during therapy, for an incidence of 3 percent. These data suggest a relatively low frequency of thrombocytopenia during heparin prophylaxis. Several suggestions are made regarding monitoring platelet counts during low-dose heparin therapy.

The occurrence of thrombocytopenia during therapeutic (full-dose) heparin adminstration is well documented, although incidence figures have varied from 0 to 30 percent. ${ }^{1-3}$ More recent analysis $^{4}$ suggests an incidence of about 5 percent. Thrombocytopenia occurs more commonly with bovine lung than with porcine intestinal heparin preparations. ${ }^{5}$ The frequency and etiology of thrombocytopenia during prophylactic (low-dose) heparin therapy have not been studied as extensively. Several prospective trials were unable to demonstrate thrombocytopenia during low-dose treatment. ${ }^{6-10}$ Also, in only 1 prospective trial ${ }^{8}$ was an attempt made to demonstrate a heparin-dependent antiplatelet antibody.

The mechanism responsible for heparin-induced thrombocytopenia is uncertain. Some evidence suggests that the development of a heparin-dependent antiplatelet antibody and subsequent comple- ment activation may be associated with thrombocytopenia induced by heparin. In addition, the plasma of patients with heparin-induced thrombocytopenia, when incubated with normal platelets and low levels of heparin, will exhibit serotonin release and platelet aggregation. Heparin-dependent platelet aggregation has been found to be a specific, although insensitive, test for heparin-induced thrombocytopenia. ${ }^{11}$

The present study was conducted to determine the frequency of thrombocytopenia during low-dose heparin therapy in the intensive care unit. In patients who became thrombocytopenic, an attempt was made to document the development of a heparin-dependent antiplatelet antibody and serotonin release reaction.

\section{Materials and methods}

Sixty-five consecutive intensive care patients receiving low-dose heparin therapy were studied prospectively after the subcutaenous administration of 5,000 units of porcine intestinal heparin every 12 hours. All patients at risk for deep vein thrombophlebitis or pulmonary embolism were studied unless specific exclusion criteria were met. These included baseline thrombocytopenia, any acute bleeding diathesis, septicemia, or the administration of heparin or chemotherapeutic agents within 6 months prior to this study.

Each patient had a baseline platelet count, prothrombin time (PT) and partial thromboplastin time (PTT) determinations, and a complete blood cell count (CBC) performed prior to the initial administration of heparin. The use of heparin was continued until the physician caring for the patient 


\begin{tabular}{|lc|}
\hline \multicolumn{2}{|l|}{ TABLE 1. PRIMARY DIAGNOSES OF PATIENTS TREATED WITH } \\
PROPHYLACTIC HEPARIN. & No. of cases \\
\hline Diagnosis & \\
\hline Medical & 4 \\
Cardiac arrhythmia & 8 \\
Acute respiratory failure & 7 \\
Malignancy & 9 \\
Congestive heart failure & \\
Acute myocardial infarct or & 18 \\
coronary insufficiency & 4 \\
Pneumonia & $\underline{4}$ \\
Neurologic disorders & 54 \\
$\quad$ Total & \\
& \\
Surgical & 5 \\
Bowel obstruction & 2 \\
Abdominal surgery & 2 \\
Gangrene & 2 \\
Fracture of femur & 11 \\
$\quad$ Total & \\
\hline
\end{tabular}

decided that prophylaxis was no longer necessary. Platelet counts and a CBC were obtained on days 2, $6,10,14$, and 21 of administration, and then weekly. If thrombocytopenia occurred (platelet count $<100,000$ /cu. mm.), heparin was discontinued, and the PT, PTT, and fibrinogen values were checked to rule out disseminated intravascular coagulation. As described by Cines and associates, ${ }^{12}$ the plasma of thrombocytopenic patients was obtained and tested for the presence of antiplatelet antibody, heparin-associated antiplatelet antibody, and ${ }^{14} \mathrm{C}$ serotonin release reaction.

Informed consent was obtained from all patients. This protocol was approved by the Institutional Review Board/Committee for the Protection of Human Subjects of the UMDNJ-SOM. Statistical comparisons between the initial and final platelet counts, leukocyte counts, hemoglobin values, and hematocrit readings for the entire group were performed using the Student's $t$ test for paired data. The initial and final platelet counts for individual disease categories were also compared by the Student's $t$ test for paired data. Comparisons between the initial platelet count for the entire group and the initial counts in individual disease categories were made using the Student's $t$ test for independent variables. Correlation coefficients were calculated from linear regression analysis, by the least squares method.

\section{Results}

A total of 65 intensive care patients ( 27 men and 38 women) were monitored during prophylactic heparin therapy. The mean age of the patients was $68.8 \pm 12.7$ years $( \pm \mathrm{SD})$. The distribution of the patients' primary diagnoses are listed in Table 1.
The mean duration of therapy was $11.1 \pm 7.7$ days (range, 3 to 45 days). During heparin therapy, an average of $8.0 \pm 3.6$ drugs were administered concurrently.

For the group as a whole, no significant changes were noted in platelet count or leukocyte count after heparin therapy (Table 2). Significant decreases were noted in both hemoglobin and hematocrit values following treatment. These decreases correlated positively, by linear regression analysis, with the duration of heparin therapy and probably were due to both phlebotomy and to nonovert gastrointestinal bleeding in intensive care patients.

Two patients developed thrombocytopenia during low-dose heparin therapy. The first was a 72year-old man whose platelet count fell to $88,000 / \mathrm{cu}$. $\mathrm{mm}$. on the third day of treatment. His plasma contained an elevated level of heparin-associated antiplatelet antibody (48.4 percent), with a normal value of less than 5 percent. In addition, the patient's plasma caused a significant ${ }^{14} \mathrm{C}$-serotonin release from normal platelets in the presence of low levels of heparin. The second thrombocytopenic patient, a 68-year-old man, had a platelet count of $57,000 / \mathrm{cu}$. mm. on day 5 of heparin treatment. Serologic studies were not obtained for this patient because he required emergency transfer to another institution for prosthetic valve placement. However, subsequent challenge of this patient with full-dose heparin resulted in profound thrombocytopenia. In both cases, platelet counts returned to normal values within 3 days of heparin discontinuation. PT, PTT, and fibrinogen assays were within normal limits when thrombocytopenia developed in both patients. No clinical evidence of bleeding or arterial thrombosis was noted in either patient at the time thrombocytopenia developed.

An attempt was made to determine whether significant reductions in platelet count occurred within specific diagnostic categories during heparin prophylaxis. As shown in Table 3, no significant differences were noted between the initial platelet count of the entire group of 65 patients and the initial platelet count for any individual disease category. In addition, no significant decrease in platelet count was noted within any diagnostic category during heparin prophylaxis. A significant increase in platelet count was noted in the surgical group during the low-dose heparin treatment. This was thought to be a reactive process due to surgical stress.

\section{Discussion}

Several prospective studies in medical patients, ${ }^{6-8}$ surgical patients, ${ }^{9,13}$ and normal individuals ${ }^{10}$ have failed to demonstrate significant throm- 
TABLE 2. RESULTS OF BLOCD STUDIES BEFORE AND ON THE LAST DAY OF LOW-DOSE HEPARIN PROPHYLAXIS IN 65 PATIENTS.

\begin{tabular}{|lcc|}
\hline $\begin{array}{l}\text { Hematologic } \\
\text { component }\end{array}$ & $\begin{array}{c}\text { Initial } \\
\text { value }\end{array}$ & $\begin{array}{c}\text { Final } \\
\text { value }\end{array}$ \\
\hline Platelets $\left(\times 10^{-3} \mathrm{~mm}^{3}\right)$ & $277 \pm 95^{*}$ & $292 \pm 135(\mathrm{NS})$ \\
Leukocytes $\left(\times 10^{-3} \mathrm{~mm}^{3}\right)$ & $11.0 \pm 4.8$ & $11.2 \pm 4.7(\mathrm{NS})$ \\
Hemoglobin $(\mathrm{gm} . / \mathrm{dl})$. & $13.0 \pm 2.2$ & $11.8 \pm 2.0(p<0.001)$ \\
Hematocrit (percent) & $39.8 \pm 6.8$ & $35.4 \pm 6.0(p<0.001)$ \\
* ( \pm standard deviation) & & \\
NS $=$ not significant by paired Student's t test & & \\
\hline
\end{tabular}

TABLE 3. PLATELET COUNTS DURING HEPARIN PROPHYLAXIS ACCORDING TO PRIMARY DIAGNOSTIC CATEGORIES.

\begin{tabular}{|c|c|c|c|}
\hline $\begin{array}{l}\text { Primary } \\
\text { diagnosis }\end{array}$ & $\begin{array}{l}\text { No. of } \\
\text { cases }\end{array}$ & $\begin{array}{l}\text { Initial value } \\
\left(\times 10^{-3} / \mathrm{mm}^{3}\right)\end{array}$ & $\begin{array}{c}\text { Final count } \\
\left(\times 10^{-3} / \mathrm{mm}^{3}\right)\end{array}$ \\
\hline Total group & 65 & $277 \pm 95$ & $292 \pm 135(\mathrm{NS}) \dagger$ \\
\hline Arrhythmia & 4 & $245 \pm 81(\mathrm{NS})^{*}$ & $265 \pm 42(\mathrm{NS})$ \\
\hline Respiratory failure & 8 & $322 \pm 74$ (NS) & $351 \pm 111(\mathrm{NS})$ \\
\hline Malignancy & 7 & $351 \pm 155(\mathrm{NS})$ & $331 \pm 223(\mathrm{NS})$ \\
\hline Congestive heart failure & 9 & $247 \pm 69(\mathrm{NS})$ & $241 \pm 91 \quad(\mathrm{NS})$ \\
\hline Acute myocardial infarct & 18 & $263+108(\mathrm{NS})$ & $255+65$ (NS) \\
\hline Pneumonia & 4 & $334 \pm 96(\mathrm{NS})$ & $299 \pm 146(\mathrm{NS})$ \\
\hline Neurologic disorders & 4 & $303 \pm 132(\mathrm{NS})$ & $386 \pm 258(\mathrm{NS})$ \\
\hline Surgical & 11 & $244 \pm 77(\mathrm{NS})$ & $307 \pm 126 p=0.04$ \\
\hline
\end{tabular}

bocytopenia during low-dose heparin administration. In the present study, a 3 percent frequency of thrombocytopenia was observed. This finding is in agreement with the combined results of three prospective trials ${ }^{14-16}$ in which 5 of 201 patients became thrombocytopenic. The reason for this discrepancy in study results undoubtedly relates to the small sample populations and shorter durations of therapy in many of the previous trials. In our study and most of the reported prospective trials (except for Saffle and coworkers' study ${ }^{10}$ ), porcine intestinal heparin was employed. Therefore, the type of heparin used does not account for the different findings.

Only one previous report demonstrated a relatively high incidence of thrombocytopenia during low-dose heparin therapy. Johnson, Lazarus, and Henry ${ }^{17}$ demonstrated thrombocytopenia in 10 of 66 patients (15 percent). However, these authors defined thrombocytopenia as a platelet count of $<150,000 / \mathrm{cu}$. mm. In their study, no patient's platelet count fell below $100,000 / \mathrm{cu}$. mm.; therefore, they considered the high incidence of thrombocytopenia to be clinically insignificant.

Including the present study, 567 patients have been prospectively studied during low-dose heparin therapy. ${ }^{6-10,13-17}$ Seven became thrombocytopenic, for a frequency of 1.2 percent. Porcine heparin was received by 542 patients, and 25 patients received beef heparin. The onset of thrombocytopenia ranged from the third to the tenth day of heparin treatment. The thrombocytopenia re- solved either spontaneously despite continued heparin therapy or within 3 to 8 days after discontinuation of treatment. No case of arterial thrombosis was described in any of the prospective studies. However, rare cases of arterial thrombosis associated with low-dose heparin-induced thrombocytopenia have been reported. ${ }^{10,17}$

Of the 7 patients who developed thrombocytopenia, 4 had resolution despite continued heparin administration. ${ }^{14,15}$ This observation has cast some doubt on whether low-dose heparin is causally related to thrombocytopenia. In our 2 cases, immunologic studies in 1 patient, and a positive heparin challenge in the second, strongly indicate true heparin-induced thrombocytopenia. Because our protocol required the discontinuation of heparin at the onset of thrombocytopenia, we cannot make any statement about spontaneous resolution based on our data. However, Chong and coauthors ${ }^{18}$ have described two types of heparininduced thrombocytopenia. The first is an early, asymptomatic type that is not associated with a heparin-dependent platelet aggregating factor. The second is a late-onset form of thrombocytopenia; it is frequently associated with thromboembolism and always associated with the development of a heparin-dependent platelet aggregating factor. It may be the early-onset type of thrombocytopenia that is related to spontaenous remission during heparin therapy. Further studies will be needed to resolve this issue.

Evidence suggests that thrombocytopenia asso- 
ciated with full-dose heparin therapy is due to platelet aggregation and sequestration. ${ }^{19-21}$ The aggregation is caused by a heparin-dependent IgG antiplatelet antibody, ${ }^{22-24}$ is complement dependent, and may be blocked by nonsteroidal, antiinflammatory agents. ${ }^{12,18,25}$ Our demonstration of a heparin-dependent antiplatelet antibody and serotonin release from normal platelets on exposure to our patient's plasma supports a similar mechanism for thrombocytopenia in patients treated with low-dose heparin.

Because of the risk of bleeding, thrombosis, and death associated with heparin-induced thrombocytopenia, several authors have recommended regular monitoring of platelet counts during heparin therapy. ${ }^{1,2}$ However, a strong argument can be made for not monitoring platelet counts during low-dose heparin therapy. First, the incidence of thrombocytopenia during prophylactic heparin therapy is low. Second, thrombocytopenia resolved despite continued low-dose heparin treatment in over half of the prospectively studied patients. Finally, no evidence of clinical bleeding or arterial thrombosis in any thrombocytopenic patient was noted in any of the prospectively studied patients. Therefore, the frequency of these effects must be even lower than thrombocytopenia during heparin prophylaxis. In light of these data, routine platelet count monitoring during low-dose heparin therapy seems unwarranted. However, if a patient on prophylactic heparin should develop bleeding, petechiae, or thrombosis, a platelet count and coagulation studies should be performed and heparin should be discontinued. If thrombocytopenia is found, the diagnosis of heparin-induced thrombocytopenia may be confirmed by the demonstration of heparin-induced platelet aggregation with low levels of heparin, ${ }^{11}$ or by ${ }^{14} \mathrm{C}$ serotonin release reaction. ${ }^{12}$ A negative test does not exclude the diagnosis.

\section{Summary}

The incidence of thrombocytopenia during lowdose heparin prophylaxis was determined in 65 intensive care patients. A low incidence of heparininduced thrombocytopenia was found. These data suggest that routine platelet count monitoring is not required during such therapy.

1. Bell, W.R., et al.: Thrombocytopenia occurring during the administration of heparin. A prospective study in 52 patients. Ann Intern Med 85:155-60, Aug 76

2. Nelson, J.C., et al.: Heparin-induced thrombocytopenia. Arch Intern Med 138:548-52, Apr 78
3. Eika, C., et al: Low incidence of thrombocytopenia during treatment with hog mucosa and beef lung heparin. Scand J Hematol 25:19-24, Jul 80 4. King, D.J., and Kelton, J.G.: Heparin-associated thrombocytopenia. Ann Intern Med 100:535-40, Apr 84

5. Bell, W.R., and Royall, R.M.: Heparin-associated thrombocytopenia. A comparison of three heparin preparations. N Engl J Med 303:902-7, 16 Oct 80

6. Malcolm, I.D., Wigmore, T.A., and Steinbrecher, U.P.: Heparin-associated thrombocytopenia. Low frequency in 104 patients treated with heparin of intestinal mucosal origin. Can Med Assoc J 120:1086-8, 5 May 79

7. Weitberg, A.B., Spremulli, E., and Cummings, F.J.: Effect of low dose heparin on platelet count. South Med J 75:190-2, Feb 82

8. Romeril, K.R., et al.: Heparin induced thrombocytopenia. Case reports and a prospective study. NZ Med J 267-9, 28 Apr 82

9. Economopoulos, T.C., et al.: Postoperative platelet function in patients on small subcutaneous doses of heparin. Acta Haematol 57: 266-71, 1977 10. Saffle, J.R., et al.: The effect of low-dose heparin therapy on serum platelet and transaminase levels. J Surg Res 28:297-305, 1980

11. Kelton, J.G., et al.: The sensitivity and specificity of the heparindependent platelet aggregating factor in patients with heparin associated thrombocytopenia (abstract). Blood 60(Suppl. 1):187A, Nov 82

12. Cines, D.B., et al.: Heparin-associated thrombocytopenia. N Engl J Med 303:788-95, 2 Oct 80

13. Rocko, J.M., et al.: The safety of low dose heparin prophylaxis. Am J Surg 135:798-800, Jun 78

14. Olin, J., and Graor, R.: Heparin-associated thrombocytopenia (letter) N Engl J Med 304:609, 5 Mar 81

15. Gallus, A.S., et al:: Heparin-associated thrombocytopenia. Case report and prospective study. Aust NZ J Med 10:25-31, Feb 80

16. Ayars, G.H., and Tikoff, G.: Incidence of thrombocytopenia in medical patients on "mini-dose" heparin prophylaxis (letter). Am Heart J 99:1816, Jun 80

17. Johnson, R.A., Lazarus, K.H., and Henry, D.H.: Heparin-induced thrombocytopenia. A prospective study. Am J Hematol 17:349-53, 1984 18. Chong, B.H., Pitney, W.R., and Castaldi, P.A.: Heparin-induced thrombocytopenia. Association of thrombotic complications with heparin-dependent IgG antibody that induces thromboxane synthesis and platelet aggregation. Lancet $2: 1246-9,4$ Dec 82

19. Fratantoni, J.C., Pollet, R., and Gralnick, H.R.: Heparin-induced thrombocytopenia. Confirmation of diagnosis with in vitro methods. Blood 45:395-401, Mar 75

20. Babcock, R.B., Dumper, C.W., and Scharfman, W.B.: Heparin-induced immune thrombocytopenia. N Engl J Med 295:237-41, 29 Jul 76

21. Wahl, T.O., Lipschitz, D.A., and Stechshulte, D.J.: Thrombocytopenia associated with antiheparin antibody. JAMA 240:2560-2, 1 Dec 78

22. Green, D., et al:: Heparin immune thrombocytopenia. Evidence for a heparin-platelet complex as the antigenic determinant. J Lab Clin Med 91:167-75, Jan 78

23. Trowbridge, A.A., et al.: Heparin-related immune thrombocytopenia. Studies of antibody-heparin specificity. Am J Med 65:277-83, Aug 78

24. Cimo, P.L., et al.: Heparin-induced thrombocytopenia. Association with platelet aggregating factor and arterial thrombosis. Am J Hematol 6:125-33, 1979

25. Chong, B.H., Grace, C.S., and Rozenberg, M.C.: Heparin-induced thrombocytopenia. Effect of heparin platelet antibody on platelets. Br J Hematol 49:531-40, Dec 81

From the Department of Internal Medicine, Pulmonary and Hematology Divisions, University of Medicine and Dentistry of New Jersey, School of Osteopathic Medicine, Stratford, New Jersey, where Dr. Morley is an assistant professor of medicine, Dr. Bannett was a fourth-year student, Dr. Giudice is a professor of medicine, and Dr. Freed is an associate professor of medicine. Dr. Morone is in the private practice of internal medicine, Wenonah, New Jersey. Dr. Bannett is now a resident in ophthalmology, Geisinger Medical Center, Danville, Pennsylvania.

Dr. Morley, Suite 3100, 301 Central Plaza, Stratford, New Jersey 08084. 


\section{In hypertension}

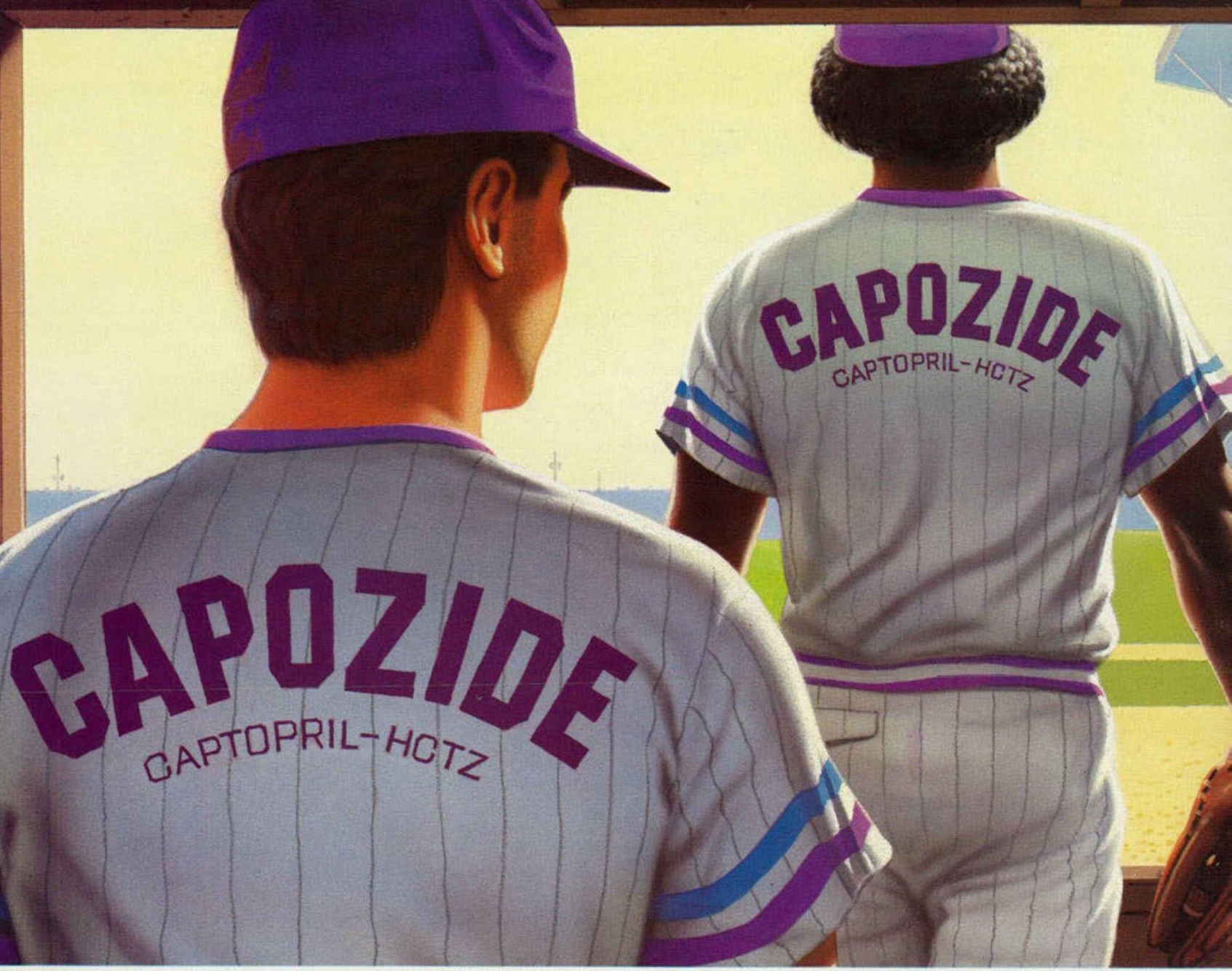

\section{THE ONLY AGENT THAT TEAMS A THIAZIDE DIURETIC WITH CAPTOPRIL}

*This fixed combination drug is not indicated for initial therapy of hypertension. It may be appropriate if the fixed combination represents the dosage as titrated to the individual patient's needs. In using CAPOZIDE, consideration should be given to the risk of neutropenia/agranulocytosis. Use special precautions in patients with impaired renal function, collagen vascular disorders, or those exposed to other drugs known to affect the white cells or immune response. Evaluation of hypertensives should always include assessment of renal function. See INDICATIONS AND USAGE, CONTRAINDICATIONS, WARNINGS, and ADVERSE REACTIONS in the brief summary. 


\section{Achieves outstanding blood pressure control with built-in potassium-sparing activity ${ }^{\star}$}

- Captopril adds exceptional control of blood pressure to that of thiazide diuretics.

- CAPOZIDE blunts potassium loss by reducing aldosterone secretion.

- CAPOZIDE enhances BP control, curbs potassium loss in one convenient tablet.

\section{No strikes on quality of life $e^{1,2}$}

- Few patient withdrawals.

- Preserves sexual function in most patients.

- Maintains general well-being during therapy.

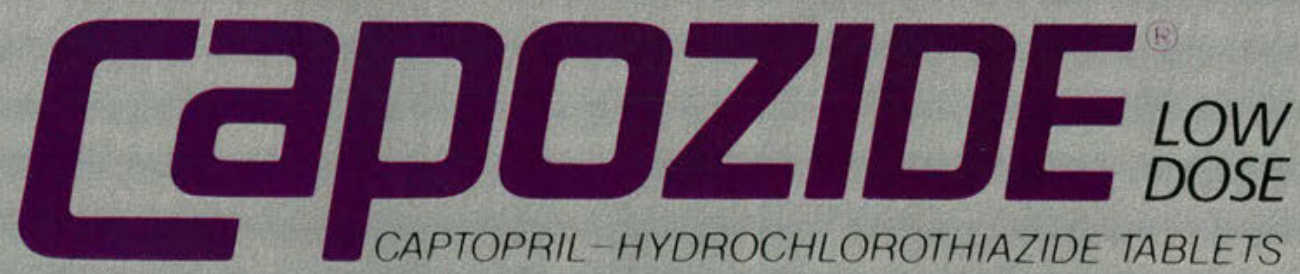




\section{CAPOZIDE}

\section{Captopril-Hydrochlorothiazide Tablets}

INDICAMONS AND USAGE:-CAPOZIDE (Captopri-Hydrochlorothiazide Tablets) is indicated for the treatment of hypertension. This fixed combination drug is not indicated for initial therapy of hypertension. If the fixed combination represents the dose titrated to the individual patiunt's needs, may be more convenient than the separate components. In using CAPOZIDE, consideration should be given to the risk of neutropenia/agranulocylosis (see WARNINGS). CAPOZIDE may be used fo patients with normal renal function, in whom the risk is relatively low. In patients with impaired renal
function, particularly those with collagen vascular disease, CAPOZIDE should be reserved for hypertensives who have either developed unacceptable side effects on other drugs, or have failed to respond satisfactorily to other drug combinations.

CONTRAINDICATION: Hydrochlorothiazide-Hydrochlorothiazide is contraindicated in patients with anuria and those who have previously demonstrated hypersensitivity to hydrochlorothiazide or other sullonamide-derived drugs.

WARNINGS: Captopril-Neutropenia/Agranulocytosis-Neutropenia $\left(<1000 / \mathrm{mm}^{3}\right)$ with myeloid hy. poplasia has resulted from use of captopril. About half of the neutropenic patients developed systemic or oral cavity infections or other leatures of the

In clinical trials in patients with hypertension who have normal renal function (serum creatinine $<1.6$ $\mathrm{mg} / \mathrm{dL}$ and no collagen vascular disease), neutropenia has been seen in one patient out of over 8,600 exposed. In patients with some degree of renal tailure (serum creatinine at least $1.6 \mathrm{mg} / \mathrm{dL}$ ) but no collagen vascular disease, the risk in clinical trials was about 1 per 500 . Doses were relatively high in these patients, particularly in view of their diminished ranal function. In patients with collagen vascular diseases (e.g., systemic lupus erythematosus, scleroderma) and impaired renal function, neutropenia occurred in $3.7 \%$ of patients in clinical trials. While none of the over 750 patients in formal clinical trials of heart failure developed neutropenia, it has occurred during subsequent clinical experience. O reported cases, about half had serum creatinine $\geq 1.6 \mathrm{mg} / \mathrm{dL}$ and more than $75 \%$ received procainamide. In heart failure, it appears that the same risk factors for neutropenia are present Neutropenia has appeared within 3 months after starting therapy, associated with myeloid hypoplasia and frequently accompanied by erythroid hypoplasia and decreased numbers of megakaryocytes (e.g. hypoplastic bone marrow and pancytopenia); anemia and thrombocytopenia were sometimes seen. Neutrophils generally returned to normal in about 2 weeks after captopril was discontinued, and serious infections were limited to clinically complex patients. About $13 \%$ of the cases of neutropenia have ended fatally, but almost all fatalities were in patients with serious illness. having collagen vascular disease, renal failure, heart failure or immunosuppressant therapy, or a combination of these complicating factors.

Evaluation of the hypertensive or heart failure patient should always include assessment of renal function. If captopril is used in patients with impaired renal function, white blood cell and differential counts should be evaluated prior to starting treatment and at approximately 2-week intervals for about 3 months, then periodically, In patients with collagen vascular disease or who are exposed to other drugs known to affect the white cells or immune response, particularly when there is impaired renal function, captopril should be used only after an assessment of benefit and risk, and then with caution. All patients treated with captopril should be told to report any signs of infection (e.g., sore throat, fever); if infection is suspected, perform counts without delay. Since discontinuation of captopril and other drugs has generally led to prompt return of the white count to normal, upon confirmation of neutropenia (neutrophil count $<1000 / \mathrm{mm}^{3}$ ) withdraw captopril and closely follow the patient's course. Proteinuria-Total urinary proteins $>1 \mathrm{~g} /$ day were seen in about $0.7 \%$ of patients on captopril. About Proteinuria-Total urinary proteins $>1 \mathrm{~g} /$ day were seen in about $0.7 \%$ of patients on captopril. About
$90 \%$ of affected patients had evidence of prior renal disease or received high doses ( $>150 \mathrm{mg} /$ day), or $90 \%$ of affected patients had evidence of prior renal disease or received high doses ( $>150 \mathrm{mg} /$ day), or uria subsided or cleared within 6 months whether or not captopril was continued. The BUN and creatiuria subsided or cleared within 6 months whether or not captopril was continued. The BUN and creati. nine were seldom altered in proteinuric patients. Since most cases of proteinuria occurred by the 8 th have urinary protein estimates (dip-stick on 1st morning urine) before therapy, and periodically thereatter. Hypotension-Excessive hypotension was rarely seen in hypertensive patients but is a possibility in severely salt/volume-depleted persons such as those treated vigorously with diuretics (see PRECAUTIONS [Drug Interactions])

Hydrochlorothiazide-Use with caution in severe renal disease. May precipitate azotemia in patients with renal disease. Cumulative effects may develop in patients with impaired renal function. Use with caution in patients with impaired hepatic function or progressive liver disease, since minor alterations of fluid and electrolyte balance may precipitate hepatic coma. Sensitivity reactions may occur in patients fluid and electrolye balance may precipitate hepatic coma. Sensitivity reactions may occur in patients
with or without a history of allergy or bronchial asthma. The possibility of exacerbation or activation of systemic lupus erythematosus has been reported.

PRECAUTONS: General: Captopril-Impaired Renal Function-Some patients with renal disease particularly those with severe renal artery stenosis, have developed increases in BUN and serum creatinine. It may be necessary to reduce captopril dosage and/or discontinue diuretic. For some of these patients, normalization of blood pressure and maintenance of adequate renal perfusion may not be possible (see DOSAGE AND ADMINISTRATION, ADVERSEREACTIONS [Altered Laboratory Findings]), Surgery/Anesthesia - It hypotension occurs during major surgery or anesthesia, and is considered due to the effects of captopril, it is correctable by volume expansion.

Hydrochlorothiazide-Observe all patients for signs of fluid or electrolyte imbalance, particularly when the patient is vomiting excessively or receiving parenteral fluids. Warning signs include dryness of mouth, thirst, weakness, lethargy, drowsiness, restlessness, muscle pains or cramps, muscular fatigue. nypotension, oliguria, tachycardia, and nausea and vomiting. Hypokalemia may develop when severe cirrhosis is present or without adequate oral electrolyte intake. Hypokalemia can sensitize or exaggerate response of the heart to the toxic effects of digitalis. Because captopril reduces the production of aldosterone, concomitant therapy with captopril reduces the diuretic-induced hypokalemia. Fewer patients may require potassium supplements and/or to

Any chloride deficit is generally mild and may not require specfic treatment, exceptions include liver disease or renal disease. Dilutional hyponatremia may occur in edematous patients in hot weather; use water restriction, rather than salt administration except when the hyponatremia is life-threatening. In actual salt depletion, replacement is the therapy of choice. Hyperuricemia may occur or frank gout may be precipitated in certain patients. Latent diabetes mellitus may become manifest. Antihypertensive effects may be enhanced in the postsympathectomy patient. Progressive renal impairment, indicated by effects may be enhanced in the postsympathectomy patient. Progressive renal impairment, indicated by
rising nonprotein nitrogen or blood urea nitrogen, requires a careful reappraisal of the necessity of therapy. Serum PBI levels may decrease. Calcium excretion is decreased. Pathologic changes in the parathyroid gland with hypercalcemia and hypophosphatemia have been observed during prolonged therapy. Laboratory Tests-Serum and urine electrolyte levels should be regularly monitored (see WARNINGS, [Captopril and Hydrochlorothiazide], also PRECAUTIONS [General, Hydrochlorothiazide]).

Drug Interactions-Captopril-Hypotension-Patients on Diuretic Therapy: Precipitous reduction of blood pressure may occasionally occur within the 1 st hour after administration of the initial captopril dose in patients on diuretics, especially those recently placed on diuretics, and those on severe dietary salt restriction or dialysis. This possibility can be minimized by either discontinuing the diuretic or increasing the salt intake about 1 week prior to initiation of captopril therapy or by initiating therapy with small doses $(6.25$ or $12.5 \mathrm{mg})$. Alternatively, provide medical supervision for at least 1 hour after the initial dose. Agents Having Vasodilator Activity: In heart failure patients, vasodilators should be administered with caution. Agents Causing Renin Release: Captopril's effect will be augmented by antihypertensive agents that cause renin release. Agents Affecting Sympathetic Activity: The sympathetic nervous syslem may be especially important in supporting blood pressure in patients receiving captopril alone or with diuretics. Beta-adrenergic blocking drugs add some further antihypertensive effect to captopril, but the overall response is less than additive. Therefore, use agents affecting sympathetic activity (e.g. ganglionic blocking agents or adrenergic neuron blocking agents) with caution. Agents increasing Serum Potassium: Give potassium sparing diuretics or potassium supplements only for documented yypokalemia, and then with caution, since they may lead to a significant increase of serum potassium. Use potassiurn-containing salt substitutes with caution. Inhibitors Of Endogenous Prostaglandin Syneffect of captopril, especially in low renin hypertension.

Hydrochlorothiazide--When administered concurrently the following drugs may interact with thiazide diuretics: Alcohol, barbiturates or narcotics-potentiation of orthostatic hypotension may occur. Antidiabetic drugs (ora/ agents and insulin) - Hyperglycemia induced by thiazides may require dosage adjustment of the antidiabetic drug. Other antihypertensive drugs-additive effect or potentiation. Potentiation occurs with ganglionic or peripheral adrenergic blocking drugs. Corticosteroids, ACTHintensified electrolye depletion, particularly hypokalemia. Preanesthetic and anesthetic agents-effects of preanesthetic and anesthetic agents may be potentiated; adjust dosage of these agents accordingly. Pressor amines (e.g. norepinephrine)-possible decreased response to pressor amines but not suttcient to preclude their use. Skeletal muscle relaxants, nondepolarizing (e.g. tubocurarine)-possible diuretic agents reduce the renal clearance of lithium and add a high risk of lithium toxicity. Refer to the package insert for lithium preparations before use of such preparations with CAPOZIDE.

Drug/Laboratory Test Interactions-Captopril-may cause a false-positive urine test for acetone. Drug/Laboratory Test Interactions -Captopril-may cause a false-positive urine test for acetone.
Hydrochlorothiazide-Discontinue thiazides before carrying out tests for parathyroid function (see Hydrochlorothiazide-Discontinue thiazides betor
PRECAUTONS [General, Hydrochlorothiazide]).

Carcinogenesis, Mutagenesis and Impairment of Fertility-Captopril-Two-year studies with doses of 50 to $1350 \mathrm{mg} / \mathrm{kg} /$ day in mice and rats failed to show any evidence of carcinogenic potential. Studies in rats have revealed no impairment of fertility.

Hydrochlorothiazide-Long-term studies in animals have not been performed to evaluate carcinogenic potential, mutagenesis, or whether this drug affects fertility in males or females.

Pregnancy: Category C-Captopril-There are no adequale and well-controlled studies in pregnant women. Embryocidal effects and craniolacial malformations were observed in rabbits. Therefore, captopril should be used during pregnancy only if the potential benefit outweighs the potential risk to the fetus. Captopril crosses the human placenta.

Hydrochlorothiazide-Studies in pregnant rats using captopril and hydrochlorothiazide individually and in combination, each agent in doses up to $1350 \mathrm{mg} / \mathrm{kg}$, falled to show evidence of embryotoxicity, fetotoxicity, or teratogenicity. There are no adequate and well-controlled studies in pregnant women. Because animal reproduction studies are not always predictive of human response, CAPOZIDE should be used during pregnancy, or in patients tikely to become pregnant, only it the potential benefit justifies the potential risk to the fetus.

Pregnancy-Nonteratogenic Effects-Hydrochlorothiazide-Thiazides cross the placental barrier ble hazards to the fetus. Hazards include fetal or neonatal jaundice, thrombocyopenia, and possibly other reported reactions.

Nursing Mothers: Both captopril and hydrochlorothiazide are excreted in human milk. A potential exists for serious adverse reactions in nursing infants from both drugs, therefore, decision whether to discontinue nursing or to discontinue therapy should take into account the importance of CAPOZIDE (CaptoprilHydrochiorothiazide Tablets) to the mother.

Pediatric Use: Safety and effectiveness in children have not been established although there is limited experience with use of captopril in children from 2 months to 15 years of age Dosage, on a weight basis, was comparable to that used in adults. CAPOZDE should be used in children only if other measures for controlling blood pressure have not been effective.

ADVERSE REACTIONS: Captopril-Rleported incidences are based on clinical trials involving approxmately 7000 patients. Renal-About 1 of 100 patients developed proteinuria (see WARNINGS). Renal insufficiency, renal failure, polyuria, oliguria, and urinary frequency in 1102 of 1000 patients. HematologicNeutropenia/agranulocytosis has occurred (see WARNINGS). Anemia, thrombocytopenia, and pancyopenia have been reported. Dermatologic-Rash (usually maculopapular, rarely urticarial), often with pruritus and sometimes with fever and eosinophilia, in aly maculopapular, rarely urticarial), onten renal status and dose), usually during the 1st 4 weeks of therapy. Pruritus, without rash, in about 2 of 100 patients. A reversible associated pemphigoid-like lesion, and photosensitsity have also been reported. patients. A reversible associated pemphigoid-like lesion, and photosensitvity have also been reported. patients-reversible on discontinuance of captopril therapy. One case of laryngeal edema reported. Fushing or pallor in 2 to 5 of 1000 patients. Cardiovascular-Hypotension may occur, see WARNINGS and PRECAUTIONS (Drug Interactions) for discussion of hypotension on intiation captopril therapy. Tachycardia, chest pain, and palpitations each in about 1 of 100 patients. Angina pectoris, myocardial infarction, Raynaud's syndrome, and congestive heart failure each in 2 to 3 of 1000 patients. DysgeusiaAbout 2 to 4 (depending on renal status and dose) of 100 patients developed a diminution or loss of taste perception; taste impairment is reversible and usually self-limited even with continued drug use ( 2 to 3 months). Gastric irritation, abdominal pain, nausea, vomiting, diarrhea, anorexia, constipation, aphthous ulcers, peptic ulcer, dizziness, headache, malaise, fatigue, insomnia, dry mouth, dyspnea, cough, alopecia, and paresthesias reported in about $0.5102 \%$ of patients but did not appear at increased trequency compared to placebo or other treatments used in controlled trials.

Hydrochlorothiazide-Gastrointestina/ System-anorexia, gastric irritation, nausea, vomiting. cramping. diarrhea, constipation, jaundice (intrahepatic cholestatic jaundice), pancreatitis, and sialadenitis. Cen tral Nervous System-dizziness, vertigo, paresthesias, headache, and xanthopsia. Hematologicleukopenia, agranulocytosis, thrombocytopenia, aplastic anemia, and hemolytic anemia. Cardiovascular-orthostatic hypotension. Hypersensitivity-purpura, photosensitivity, rash, urticaria, necrotizing angitis (vascultis; cutaneous vasculitis), fever, respiratory distress including pneumonitis, and anaphylactic reactions. Other-hyperglycemia, glycosuria, hyperunicemia, muscle spasm, weakness, restlessness, and tran

reduce or withdraw therapy.

Altered Laboratory Findings: Elevations of liver enzymes have been noted in a few patients but no causal relationship to captopril use has been established. Rare cases of cholestatic jaundice and hepatocellular iniury with or without secondary cholestasis have been reported in association with captopril administration. A transient elevation of BUN and serum creatinine may occur, especially in patients who are volume-depleted or who have renovascular hypertension. In instances of rapid reduction of longstanding or severely elevated blood pressure, the glomerular filtration rate may decrease ransienty, also resulting in transient rises in serum creatinine and BUN. Small increases in the serum potassium concentration frequently occur, especially in patients with renal impairment (see PRECAUTIONS) OVERDOSAGE: Captopril-Primary concern is correction of hypotension. Volume expansion with an IV infusion of normal saline is the treatment of choice for restoration of blood pressure. Captopril may be infusion of normal saline is the treatment of choice for

Hydrochlorothiazide-In addition to diuresis, overdosage of thiazides may produce varying degrees of ethargy which may progress to coma within a few hours, with minimal respiratory and cardiovascular depression and without evidence of serum electrolyte changes or dehydration. The mechanism of thiazide-induced CNS depression is unknown. Gastrointestinal irritation and hypermotility may occur ransitory increase in BUN has been reported, and serum electrolyte changes may occur, especially in patients with impaired renal function. In addition to gastric lavage and supportive therapy for stupor of coma, symptomatic treatment of gastrointestinal eflects may be needed. Degree of removal by hemodialysis has not been clearly established. Measures to maintain hydration, electrolye balance, espiration, and cardiovascular and renal function should be instituted.

DOSAGE AND ADMINISTRATION: DOSAGE MUST BE INDNIDUALZED (SEE INDICATIONS AND US AGE). CAPOZIDE (Captopril-Hydrochlorothiazide Tablets) should be taken one hour before meals CAPOZIDE may be dosed bid or tid. Because captopril and hydrochlorothiazide are excreted prima by the kidneys, dosage adjustments are recommended for patients with impaired renal function. Available in tablets of $25 \mathrm{mg}$ captopril combined with $15 \mathrm{mg}$ hydrochlorothiazide, $25 \mathrm{mg}$ captopri combined with $25 \mathrm{mg}$ hydrochlorothiazide, $50 \mathrm{mg}$ captopril combined with $15 \mathrm{mg}$ hydrochlorothiazide, and $50 \mathrm{mg}$ captopril combined with $25 \mathrm{mg}$ hydrochlorothiazide in bottles of 100 . (J4-005C)

References: 1. Data on file, University of Connecticut. 2. Croog SH, Levine S, Testa MA, et al: N Engl J Med 314(26):1657-1664, 1986.

\section{(III)} HELPING TO IMPROVE THE QUALITY OF YOUR LIFE SQUIBB 